\title{
Incorporation of Generalized Uncertainty Principle into Lifshitz Field Theories
}

\author{
Mir Faizal $^{1}$ and Barun Majumder ${ }^{2}$ \\ ${ }^{1}$ Department of Physics and Astronomy, \\ University of Waterloo, Waterloo, \\ Ontario N2L 3G1, Canada \\ f2mir@uwaterloo.ca \\ ${ }^{2}$ Indian Institute of Technology Gandhinagar, \\ Ahmedabad, 382424, India \\ barunbasanta@iitgn.ac.in
}

\begin{abstract}
In this paper, we will incorporation the generalized uncertainty principle into field theories with Lifshitz scaling. We will first construct both bososnic and fermionic theories with Lifshitz scaling based on generalized uncertainty principle. After that we will incorporate the generalized uncertainty principle into an non-abelian gauge theory with Lifshitz scaling. We will observe that even though the action for this theory is non-local, it is invariant under local gauge transformations. We will also perform the stochastic quantization of this Lifshitz fermionic theory based generalized uncertainty principle.
\end{abstract}

\section{Introduction}

The classical picture of spacetime breaks down in most approaches to quantum gravity. This is due to the fluctuations in the geometry being of order one at Planck scale. Thus, the picture of spacetime as a continuous differential manifold cannot be valid below Planck length. Furthermore, the existence of a minimum length scale is also a feature of string theory 1 1 - 5. In fact, in loop quantum gravity the existence of minimum length turns big bang into a big bounce [6. However, the existence of minimum length is not consistent with conventional uncertainty principle, which states that one can measure length with arbitrary accuracy, if one takes no measurement of momentum 7. 21. Thus, the uncertainty principle has to be modified if one wants to incorporate the existence of minimum length scale. These considerations have led to a modification of the Heisenberg uncertainty principle, which in turn has led to a modification of the Heisenberg algebra. It may be noted that the implications of this modified uncertainty principle for quantum field theory have also been studied 22]-24]. In this paper, we analyse a quantum field theory based on generalized uncertainty with Lifshitz scaling. Lifshitz field theories are quantum field theories based on an anisotropic scaling between space and time. 
Lifshitz theories were first introduced in condensed matter physics to model quantum criticality [25]-28]. In fact, a Fermi-surface-changing Lifshitz transition occurs for some heavy fermion compounds 29. The location of this Fermi-surface-changing Lifshitz transition is influenced by carrier doping. Due to strong correlations, a heavy band does not shift rigidly with the chemical potential and the actual shift is determined by the interplay of heavy and additional light bands crossing the Fermi level. Furthermore, meta-magnetic transitions in models for heavy fermions has also been analysed using doped Kondo lattice model in two dimensions 30. Some heavy fermion metals displays a field-driven quantum phase transition due to a breakdown of the Kondo effect [31-32. Many of the properties have been described by a Zeeman-driven Lifshitz transition of narrow heavy fermion bands 33. Materials that cannot be described with the local dielectric response have been described by a generalization of the usual Lifshitz theory [34. In fact, the temperature correction to the Casimir-Lifshitz free energy between two parallel plates made of dielectric material, possessing a constant conductivity at low temperatures, has been calculated [35]. Lifshitz theory have also been used for calculating the van der Waals and Casimir interaction between graphene and a material plate, graphene and an atom or a molecule, and between a single-wall carbon nanotube and a plate [36. In this model the reflection properties of electromagnetic oscillations on graphene are governed by the specific boundary conditions imposed on the infinitely thin positively charged plasma sheet, carrying a continuous fluid with some mass and charge density.

Fermionic retarded Green's function with $z=2$ has been studied at finite temperature and finite chemical potential [37. Here the usual Lifshitz geometry was replaced by a Lifshitz black hole. Hawking radiation for Lifshitz fermions has also been studied [38. Fermionic theories with $z=2$ Lifshitz scaling have also been constructed using a non-local differential operator 39]. This nonlocal differential operator is defined using harmonic extension of a compactly supported function [40-44. It appears as a map from the Dirichlet-type problem to the Neumann type problem. It may be noted that fermionic theories with $z=3$ have also been studied [45]-46]. It has been demonstrated that Nambu-Jona-Lasinio type four-fermion coupling at the $z=3$ Lifshitz fixed point in four dimensions is asymptotically free and generates a mass scale [47. In this paper, we will study both bososnic and fermionic Lifshitz field theory, consistent with generalized uncertainty principle. We will also study the gauge symmetry for these theories. It may also be noted that another interesting deformation of quantum mechanics comes from stochastic quantization [51][54]. Stochastic quantization has provided a powerful framework for analysing bosonic theories with Lifshitz scaling [55]-56. In fact, effect of ohmic noise on the non-Markovian spin dynamics resulting in Kondo-type correlations have been studied using stochastic quantization [57] In this paper, we will analyse the stochastic quantization of Lifshitz Dirac equation with minimum length.

\section{Generalized Uncertainty Principle}

In the Lifshitz field theories the scaling is usually taken as $x \rightarrow b x$ and $t \rightarrow b^{z} t$, where $b$ is called the scaling factor and $z$ is called the degree of anisotropy. For $z=1$, this reduces to the usual conformal transformation. In this paper, we 
will analyze the Lifshitz theories with $z=2$. The Lifshitz action for a bosonic field with $z=2$, can be written as [39]

$$
S_{b}=\frac{1}{2} \int d^{d+1} x\left(\phi \partial^{0} \partial_{0} \phi-\kappa^{2} \phi\left(\partial^{i} \partial_{i}\right)^{2} \phi\right) .
$$

The Lifshitz theories are unitarity becuase they contain no higher order temporal derivatives. So, we will leave the temporal part of the Lifshitz action for a bosonic field undeformed. However, we will deform its spatial part, to make it consistent with the existence of a minimum measurable length 21- 22. The Heisenberg uncertainty principle is not consistent with the existence of a minimum measurable length, as according to it, we can measure length up to arbitrary accuracy, if we do not measure the momentum. So, to accommodate the existence of a minimum measurable length scale, the Heisenberg uncertainty principle has to be modified to the generalized uncertainty principle. The generalized uncertainty principle can derived from a deformed Heisenberg algebra. The deformation of the Heisenberg algebra in turn deforms the coordinate representation of the momentum operator, and this deforms the Laplacian to $\partial^{i} \partial_{i} \rightarrow \partial^{i} \partial_{i}\left(1-\beta \partial^{j} \partial_{j}\right)$ [22. Now using this definition of the deforms the Laplacian, the deformed Lifshitz action can be written as

$$
S_{b}=\frac{1}{2} \int d^{d+1} x\left(\phi \partial^{0} \partial_{0} \phi-\kappa^{2} \phi\left[\partial^{i} \partial_{i}\left(1-\beta \partial^{j} \partial_{j}\right)\right]^{2} \phi\right) .
$$

Here we have to promote that parameter $\beta$ to a background field, such that it scales as $\beta \rightarrow b^{2} \beta$. This ensures that theory still has Lifshitz scaling after it has been deformed by the generalized uncertainty principle. It may be noted that it is common to promote parameters in conformal field theories to background field in this way [48-49]. These background fields have scaling properties that ensures the conformal invariance of the deformed theory. Now we can write this action as

$$
S_{b}=\frac{1}{2} \int d^{d+1} x\left(\phi \partial^{0} \partial_{0} \phi-\kappa^{2} \partial^{i} \phi \mathcal{T}_{\partial}^{2}\left(1-\beta \partial^{j} \partial_{j}\right)^{2} \partial_{i} \phi\right),
$$

where $\mathcal{T}_{\partial}=\sqrt{-\partial^{i} \partial_{i}}$. It may be noted that the non-local differential operator $\mathcal{T}_{\partial}$ is crucial in constructing the fermionic action with Lifshitz scaling.

Even though this operator is non-local it can be effectively viewed as a local operator, by using the theory of harmonic extension of functions from $R^{d}$ to $R^{d} \times(0, \infty)\left[39\right.$ - [44]. Thus, we can define $\mathcal{T}_{\partial}$ by its action on functions $f: R^{d} \rightarrow R$, such that its harmonic extension $u: R^{d} \times(0, \infty) \rightarrow R$ satisfies, $\mathcal{T}_{\partial} f(x)=-\left.\partial_{y} u(x, y)\right|_{y=0}$. This is because if we start with a function $f: R^{d} \rightarrow$ $R$, and find a harmonic function $u: R^{d} \times(0, \infty) \rightarrow R$, such that its restriction to $R^{d}$ coincides with the original function $f: R^{d} \rightarrow R$, then it is possible to find $u$ by solving a Dirichlet problem. This Dirichlet problem can be expressed in terms of the Laplacian in $R^{d+1}$, which is denoted by $\partial_{d+1}^{2}$. So, for $x \in R^{d}$ and $y \in R$, we have, $u(x, 0)=\phi(x)$ and $\partial_{d+1}^{2} u(x, y)=0$. In fact, for a smooth function $C_{0}^{\infty}\left(R^{d}\right)$, there is a unique harmonic extension $u \in C^{\infty}\left(R^{d} \times(0, \infty)\right)$. Now as $\mathcal{T}_{\partial} \phi(x)$ also has a harmonic extension to $R^{d} \times(0, \infty)$, we can obtain the following result, $\mathcal{T}_{\partial}^{2} \phi(x)=\left.\partial_{y}^{2} u(x, y)\right|_{y=0}=-\left.\partial^{i} \partial_{i} u(x, y)\right|_{y=0}$. Thus, it is possible to define $\mathcal{T}_{\partial}=\sqrt{-\partial^{i} \partial_{i}}$, because $\mathcal{T}_{\partial}^{2} \phi(x)=-\partial^{i} \partial_{i} \phi(x)$. So, we can write $\mathcal{T}_{\partial} \exp i k x=|k| \exp i k x$, because $\mathcal{T}_{\partial}^{2} \exp i k x=|k|^{2} \exp i k x$. Furthermore, if we 
start with two fields $\phi_{1}(x)$ and $\phi_{2}(x)$, such that $u_{1}(x, y)$ and $u_{2}(x, y)$ are their harmonic extensions to $C=R^{d} \times(0, \infty)$, and both these harmonic extensions vanish for $|x| \rightarrow \infty$ and $|y| \rightarrow \infty$, then we can write [50]

$$
\int_{C} d^{d} x d y u_{1}(x, y) \partial_{n+1}^{2} u_{2}(x, y)-\int_{C} d^{d} x d y u_{2}(x, y) \partial_{n+1}^{2} u_{1}(x, y)=0 .
$$

Thus, we get the following expression

$$
\left.\int_{R^{d}} d^{d} x\left(u_{1}(x, y) \partial_{y} u_{2}(x, y)-u_{2}(x, y) \partial_{x} u_{1}(x, y)\right)\right|_{y=0}=0 .
$$

This can now be written in terms of $\phi_{1}(x)$ and $\phi_{2}(x)$ as

$$
\int_{R^{d}} d^{d} x\left(\phi_{1}(x) \partial_{y} \phi_{2}(x)-\phi_{2}(x) \partial_{x} \phi_{1}(x)\right)=0 .
$$

So, the operator $\mathcal{T}_{\partial}$ can be moved from $\phi_{2}(x)$ to $\phi_{1}(x)$,

$$
\int_{R^{d}} d^{d} x \phi_{1}(x) \mathcal{T}_{\partial} f \phi_{2}(x)=\int_{R^{d}} d^{d} x \phi_{2}(x) \mathcal{T}_{\partial} \phi_{1}(x) .
$$

Now the Lifshitz bososnic action, consistent with generalized uncertainty principle, can also be written as

$$
S_{b}=\frac{1}{2} \int d^{d+1} x \partial^{\mu} \phi G_{\mu \nu} \partial^{\nu} \phi,
$$

where $G_{\mu \nu}$ can be written as

$$
G_{\mu \nu}=\left(\begin{array}{cc}
I_{1 \times 1} & 0_{1 \times d} \\
0_{d \times 1} & -\kappa^{2} \mathcal{T}_{\partial}^{2}\left(1-\beta \partial^{j} \partial_{j}\right)^{2} I_{d \times d}
\end{array}\right) .
$$

This equation can now be regarded as defining a scalar product for vector fields, such that for any two vectors $V$ and $W$, we have

$$
(V(x), W(x))=\int d^{d+1} x\left(V_{0} W_{0}-\kappa^{2} V_{i} \mathcal{T}_{\partial}^{2}\left(1-\beta \partial^{j} \partial_{j}\right) W_{i}\right) .
$$

The under group of isometries this inner product remains invariant. So, we can write, $(\Lambda(V), \Lambda(W))=(V, W)$. Thus, we can write $\Lambda_{0 \mu} \Lambda_{0 \nu}-\kappa^{2} \Lambda_{i \mu} \Lambda_{i \nu} \mathcal{T}_{\partial}(1-$ $\left.\beta \partial^{j} \partial_{j}\right)=G_{\mu \nu}$. From this we can infer that $\Lambda_{00}=\Lambda_{i 0}=\Lambda_{0 i}=0$ and $\Lambda_{i}^{k} \Lambda_{k j}=$ $\delta_{i j}$. Now a set of local gamma matrices can be defined, such that $\left\{\Gamma_{\mu}, \Gamma_{\nu}\right\}=$ $2 G_{\mu \nu}$. Furthermore, an appropriate choice for these local gamma matrices is $\Gamma_{0}=\gamma_{0}$ and $\Gamma_{i}=\kappa \mathcal{T}_{\partial}\left(1-\beta \partial^{j} \partial_{j}\right) \gamma_{i}$, where $\left\{\gamma_{a}, \gamma_{b}\right\}=2 \eta_{a b}$. We can thus define a fermionic Lifshitz operator as $\Gamma^{\mu} \partial_{\mu}=\gamma^{0} \partial_{0}+\kappa \gamma^{i} \mathcal{T}_{\partial}\left(1-\beta \partial^{j} \partial_{j}\right) \partial_{i}$. We observe that $\Gamma^{\mu} \partial_{\mu} \Gamma^{\nu} \partial_{\nu}=\partial^{0} \partial_{0}-\kappa^{2}\left[\partial^{i} \partial_{i}\left(1-2 \beta \partial^{j} p \partial_{j}\right)\right]^{2}$. The Lifshitz action for a massless fermionic field can be written as

$$
\begin{aligned}
S_{f} & =\frac{1}{2} \int d^{d+1} x \bar{\psi}\left(\Gamma^{\mu} \partial_{\mu}\right) \psi \\
& =\frac{1}{2} \int d^{d+1} x \bar{\psi}\left(\gamma^{0} \partial_{0}+\gamma^{i} \kappa \mathcal{T}_{\partial}\left(1-\beta \partial^{j} \partial_{j}\right) \partial_{i}\right) \psi .
\end{aligned}
$$




\section{$3 \quad$ Gauge Symmetry}

In this section, we will analyse gauge theories with Lifshitz corresponding to generalized uncertainty principle. We note that if the covariant derivative is gauge covariant, then so, is any function of the covariant derivative. We will construct a covariant derivative from using a non-abelian gauge field $A_{\mu}=A_{\mu}^{A} T_{A}$, where $\left[T_{A}, T_{B}\right]=i f_{A B}^{C} T_{C}$. Now if $\psi \rightarrow U \psi$, then we should have $D_{\mu} \psi \rightarrow U D_{\mu} \psi$. We can construct a covariant derivative with this transformation property if, we assume that the gauge field transforms as $A_{\mu} \rightarrow i U D_{\mu} U^{-1}$ and define the gauge covariant derivative as, $D_{\mu}=\partial_{\mu}+i A_{\mu}$. This is because now the covariant derivative will transform as

$$
D_{\mu} \rightarrow U D_{\mu} U^{-1}
$$

and so, $D_{\mu} \psi \rightarrow U D_{\mu} \psi$, if $\psi \rightarrow U \psi$. Now any function of the covariant derivative is also gauge covariant. So, if we take a general function of $D_{\mu}, f\left(D^{\nu} D_{\nu}\right) D_{\mu}$, then it transforms as

$$
f\left(D^{\nu} D_{\nu}\right) D_{\mu} \rightarrow U f\left(D^{\nu} D_{\nu}\right) D_{\mu} U^{-1}
$$

such that, $f\left(D^{\nu} D_{\nu}\right) D_{\mu} \psi \rightarrow U f\left(D^{\nu} D_{\nu}\right) D_{\mu} \psi$.

We can now use different $f\left(D^{\nu} D_{\nu}\right)$, for the spatial and temporal part of the covariant derivative. Now we define $f_{1}\left(D^{\nu} D_{\nu}\right)$ to be the function for the temporal part of the derivative and $f_{2}\left(D^{\nu} D_{\nu}\right)$ to be the function for the spatial part of the derivative. The theory has Lifshitz scaling, if we choose

$$
\begin{aligned}
f_{1}\left(D^{\nu} D_{\nu}\right) D_{0} & =D_{0}, \\
f_{2}\left(D^{\nu} D_{\nu}\right) D_{i} & =\kappa \mathcal{T}_{D} D_{i},
\end{aligned}
$$

where $\mathcal{T}_{D}=\sqrt{-D^{i} D_{i}}$. The covariant derivative will still transform as

$$
\begin{aligned}
D_{0} & \rightarrow U D_{0} U^{-1}, \\
\kappa \mathcal{T}_{D} D_{i} & \rightarrow U \kappa \mathcal{T}_{D} D_{i} U^{-1} .
\end{aligned}
$$

Here we have to again assumed that $\beta$ is background field which scales like $\beta \rightarrow b^{2} \beta$. [48]- 49]. This ensures that the theory constructed also has Lifshitz scaling. However, we also want a theory that will correspond to generalized uncertainty principle. In particular the matter part of the Lagrangian should reduce to the Lagrangian derived in the previous section, if we set all the gauge field to zero. Thus, we re-define $f_{1}\left(D^{\nu} D_{\nu}\right)$ and $f_{2}\left(D^{\nu} D_{\nu}\right)$ as

$$
\begin{aligned}
f_{1}\left(D^{\nu} D_{\nu}\right) D_{0} & =D_{0}, \\
f_{2}\left(D^{\nu} D_{\nu}\right) D_{i} & =\kappa \mathcal{T}_{D}\left(1-\beta D^{j} D_{j}\right) D_{i} .
\end{aligned}
$$

It may be noted that the covariant derivative will still transforms as

$$
\begin{aligned}
D_{0} & \rightarrow U D_{0} U^{-1} \\
\kappa \mathcal{T}_{D}\left(1-\beta D^{j} D_{j}\right) D_{i} & \rightarrow U \kappa \mathcal{T}_{D}\left(1-\beta D^{j} D_{j}\right) D_{i} U^{-1} .
\end{aligned}
$$

So, we can now write the final action as

$$
S=\frac{1}{2} \int d^{d+1} x \operatorname{Tr}\left[\bar{\psi}\left(\gamma^{0} D_{0}+\gamma^{i} \kappa \mathcal{T}_{D}\left(1-\beta D^{j} D_{j}\right) D_{i}\right) \psi\right] .
$$


Now the temporal part of this action is invariant under local gauge transforms because, $\operatorname{Tr}\left[\bar{\psi}\left(\gamma^{0} D_{0}\right) \psi\right] \rightarrow \operatorname{Tr}\left[\bar{\psi} U^{-1} U\left(\gamma^{0} D_{0}\right) U^{-1} U \psi\right]=\operatorname{Tr}\left[\bar{\psi}\left(\gamma^{0} D_{0}\right) \psi\right]$, and the spatial part of this action is also invariant under local gauge transforms because, $\operatorname{Tr}\left[\bar{\psi}\left(\gamma^{i} \kappa \mathcal{T}_{D}\left(1-\beta D^{j} D_{j}\right) D_{i}\right) \psi\right] \rightarrow \operatorname{Tr}\left[\bar{\psi} U^{-1} U\left(\gamma^{i} \kappa \mathcal{T}_{D}\left(1-\beta D^{j} D_{j}\right) D_{i}\right) U^{-1} U \psi\right]=$ $\operatorname{Tr}\left[\bar{\psi}\left(\gamma^{i} \kappa \mathcal{T}_{D}\left(1-\beta D^{j} D_{j}\right) D_{i}\right) \psi\right]$. So, even though this action is non-local, it is invariant under local gauge transformations, $A_{\mu} \rightarrow i U D_{\mu} U^{-1}$.

It may be noted that we can now define a gauge field tensor for this theory as

$$
\begin{aligned}
& F_{i 0}=-i\left[D_{0}, \kappa \mathcal{T}_{D}\left(1-\beta D^{j} D_{j}\right) D_{i}\right] \\
& F_{i j}=-i\left[\kappa \mathcal{T}_{D}\left(1-\beta D^{k} D_{k}\right) D_{i}, \kappa \mathcal{T}_{D}\left(1-\beta D^{l} D_{l}\right) D_{j}\right] .
\end{aligned}
$$

It transforms as

$$
\begin{aligned}
F_{i 0} \rightarrow \quad & -i\left[U D_{0} U^{-1}, U \kappa \mathcal{T}_{D}\left(1-\beta D^{j} D_{j}\right) D_{i} U^{-1}\right] \\
& =U F_{i 0} U^{-1} \\
F_{i j} \rightarrow & -i\left[U \kappa \mathcal{T}_{D} U^{-1}\left(1-\beta U D^{k} U^{-1} U D_{k} U-1\right) U D_{i} U^{-1}\right. \\
& \left.U \kappa \mathcal{T}_{D} U^{-1}\left(1-\beta U D^{l} U^{-1} U D_{l}\right) U^{-1} U D_{j} U^{-1}\right] \\
& =U F_{i j} U^{-1}
\end{aligned}
$$

Now we can write the action for the gauge part of the action as follows,

$$
S_{g}=-\frac{1}{4} \int d^{d+1} x \operatorname{Tr}\left[F^{\mu \nu} F_{\mu \nu}\right]
$$

It may be noted that even thought this action is non-local, it is invariant under local gauge transformations, $A_{\mu} \rightarrow i U D_{\mu} U^{-1}$, because, $\operatorname{Tr}\left[F^{\mu \nu} F_{\mu \nu}\right] \rightarrow$ $\operatorname{Tr}\left[U F^{\mu \nu} U^{-1} U F_{\mu \nu} U^{-1}\right]=\operatorname{Tr}\left[F^{\mu \nu} F_{\mu \nu}\right]$. Now we can write the gauge fixing term for this theory,

$$
S_{g h}=\int d^{d+1} x \operatorname{Tr}\left[b \partial^{0} A_{0}-b \kappa \partial^{i} \mathcal{T}_{\partial}\left(1-\beta \partial^{j} \partial_{j}\right) A_{i}\right] .
$$

The ghost term for corresponding to this gauge fixing term, can be written as

$$
S_{g f}=\int d^{d+1} x \operatorname{Tr}\left[\bar{c} \partial^{0} D_{0} c-\kappa^{2} \bar{c} \partial^{i} \mathcal{T}_{\partial}\left(1-\beta \partial^{j} \partial_{j}\right) D_{i}\left(1-\beta D^{k} D_{k}\right) \mathcal{T}_{D} c\right] .
$$

\section{Stochastic Quantization}

In this section, the stochastic quantization of the Lifshitz fermionic theory based generalized uncertainty principle will be analysed. To perform this analysis an an extra fictitious time variable $\tau$ will be introduced, such that $\psi(x) \rightarrow \psi(x, t)$, and $\bar{\psi}(x) \rightarrow \bar{\psi}(x, t)$ [51- [54]. We will also use an appropriate Kernel $K(x, y)$ to ensure the relaxation process is such that the systems will approach equilibrium as $\tau \rightarrow 0$. The anticommuting fermionic Gaussian noise, $\eta(x, \tau)$ and $\eta(x, \tau)$, will satisfy

$$
\begin{aligned}
\langle\eta(x, \tau)\rangle & =0 \\
\langle\bar{\eta}(x, \tau)\rangle & =0 \\
\left\langle\eta\left(x^{\prime}, \tau^{\prime}\right) \bar{\eta}(x, \tau)\right\rangle & =2 \delta\left(\tau-\tau^{\prime}\right) K\left(x, x^{\prime}\right) \delta^{4}\left(x-x^{\prime}\right) .
\end{aligned}
$$


The Langevin equations for the Lifshitz fermionic theory based on the generalized uncertainty principle can be written as

$$
\begin{aligned}
& \frac{\partial \psi(x, \tau)}{\partial \tau}=-\int d^{4} y K(x, y) \frac{\delta S[\psi, \bar{\psi}]}{\delta \bar{\psi}}+\eta(x, \tau), \\
& \frac{\partial \bar{\psi}(x, \tau)}{\partial \tau}=\int d^{4} y \frac{\delta S[\psi, \bar{\psi}]}{\delta \psi} K(x, y)+\bar{\eta}(x, \tau) .
\end{aligned}
$$

Here the action for the Lifshitz fermionic theory based generalized uncertainty principle is given by

$$
S=\frac{1}{2} \int d^{d+1} x \operatorname{Tr}\left[\bar{\psi}\left(\gamma^{0} D_{0}+\gamma^{i} \kappa \mathcal{T}_{D}\left(1-\beta D^{j} D_{j}\right) D_{i}\right) \psi\right] .
$$

It may be noted that only $\psi$ and $\bar{\psi}$ are the dynamical variables, as we are analysing the system on a fixed background. The partition function for the Lifshitz fermionic theory based generalized uncertainty principle, can be expressed as

$$
Z=\int D \bar{\eta} D \eta \exp \left(-\frac{1}{2} \int d^{4} x d^{4} y d \tau \bar{\eta}(x, \tau) K^{-1}(x, y) \eta(y, \tau)\right) .
$$

This partition function can now be expressed as

$$
\begin{aligned}
Z= & \int D \bar{\psi} D \psi \operatorname{det}\left[\frac{\delta \bar{\eta}}{\delta \bar{\psi}}\right]^{-1} \operatorname{det}\left[\frac{\delta \eta}{\delta \psi}\right]^{-1} \\
& \times \exp \left(-\frac{1}{2} \int d^{4} x d^{4} y d \tau \bar{\eta}(x, \tau) K^{-1}(x, y) \eta(y, \tau)\right) .
\end{aligned}
$$

Finally, using Langevin equations, we obtain,

$$
\begin{aligned}
Z= & \int D \bar{\psi} D \psi \operatorname{det}\left[K^{-1} \frac{\partial}{\partial \tau}-\frac{\delta^{2} S[\bar{\psi}, \psi]}{\delta \bar{\psi} \delta \psi}\right]^{-1} \operatorname{det}\left[K^{-1} \frac{\partial}{\partial \tau}+\frac{\delta^{2} S[\bar{\psi}, \psi]}{\delta \psi \delta \bar{\psi}}\right]^{-1} \\
& \times \exp \left(-\frac{1}{2} \int d^{4} x d^{4} y d \tau\left[\frac{\partial \bar{\psi}}{\partial \tau} K^{-1}-\frac{\delta S[\bar{\psi}, \psi]}{\delta \psi}\right]\right. \\
& \left.\times\left[\frac{\partial \psi}{\partial \tau}+K \frac{\delta S[\bar{\psi}, \psi]}{\delta \bar{\psi}}\right]\right)
\end{aligned}
$$

Here the determinant is defined as the regularized product of eigenvalues. This is done by using ghost fields $\left(c_{1}, \bar{c}_{1}, c_{2}, \bar{c}_{2}\right)$, and writing [58],

$$
\begin{aligned}
& \bar{c}_{1} \operatorname{det}\left[K^{-1} \frac{\partial}{\partial \tau}-\frac{\delta^{2} S}{\delta \psi \delta \bar{\psi}}\right] c_{1}=\lambda \bar{c}_{1} c_{1}, \\
& \bar{c}_{2} \operatorname{det}\left[K^{-1} \frac{\partial}{\partial \tau}-\frac{\delta^{2} S}{\delta \psi \delta \bar{\psi}}\right] c_{2}=\lambda \bar{c}_{2} c_{2} .
\end{aligned}
$$

So, we get

$$
\begin{aligned}
\mathcal{L}_{e f f}= & \frac{1}{2} \frac{\partial \bar{\psi}}{\partial \tau} K^{-1} \frac{\partial \psi}{\partial \tau}-\frac{1}{2} \frac{\delta S[\bar{\psi}, \psi]}{\delta \psi} K \frac{\delta S[\bar{\psi}, \psi]}{\delta \bar{\psi}} \\
& +\bar{c}_{1}\left[K^{-1} \frac{\partial}{\partial \tau}-\frac{\delta^{2} S[\bar{\psi}, \psi]}{\delta \bar{\psi} \delta \psi}\right] c_{1} \\
& +\bar{c}_{2}\left[K^{-1} \frac{\partial}{\partial \tau}+\frac{\delta^{2} S[\bar{\psi}, \psi]}{\delta \psi \delta \bar{\psi}}\right] c_{2} .
\end{aligned}
$$


The auxiliary fields $\bar{F}$ and $F$ are introduced to write the partition function as [59]-60]

$$
Z=\int D \bar{\psi} D \psi D c_{1} D \bar{c}_{1} D c_{2} D \bar{c}_{2} D F D \bar{F} \exp \left(-\int d^{4} x e \mathcal{L}_{e f f}\right),
$$

where

$$
\begin{aligned}
\mathcal{L}_{e f f}= & 2 \bar{F} K^{-1} F+i\left[\frac{\partial \bar{\psi}}{\partial \tau} K^{-1}-\frac{\delta S[\bar{\psi}, \psi]}{\delta \psi}\right] F \\
& +i \bar{F}\left[\frac{\partial \psi}{\partial \tau}+K \frac{\delta S[\bar{\psi}, \psi]}{\delta \bar{\psi}}\right]+\bar{c}_{1}\left[K^{-1} \frac{\partial}{\partial \tau}-\frac{\delta^{2} S[\bar{\psi}, \psi]}{\delta \bar{\psi} \delta \psi}\right] c_{1} \\
& +\bar{c}_{2}\left[K^{-1} \frac{\partial}{\partial \tau}+\frac{\delta^{2} S[\bar{\psi}, \psi]}{\delta \psi \delta \bar{\psi}}\right] c_{2} .
\end{aligned}
$$

This action can be written using the superfield formalism. Thus, complex superfields superfields $\Omega(x, \tau, \theta, \bar{\theta})$ and $\Omega(x, \tau, \theta, \bar{\theta})$, are defined as

$$
\begin{aligned}
& \Omega(x, \tau, \theta, \bar{\theta})=\psi(x, \tau)+\bar{\theta} c_{1}(x, \tau)+\bar{c}_{2}(x, \tau) \theta+i \theta \bar{\theta} F(x, \tau) \\
& \bar{\Omega}(x, \tau, \theta, \bar{\theta})=\bar{\psi}(x, \tau)+\bar{\theta} c_{2}(x, \tau)+\bar{c}_{1}(x, \tau) \theta+i \bar{\theta} \theta \bar{F}(x, \tau) .
\end{aligned}
$$

The following superderivatives and supercharges are also defined,

$$
\begin{aligned}
D & =\frac{\partial}{\partial \bar{\theta}}-\theta \frac{\partial}{\partial \tau}, & Q & =\frac{\partial}{\partial \bar{\theta}}, \\
\bar{Q} & =\frac{\partial}{\partial \theta}+\bar{\theta} \frac{\partial}{\partial \tau}, & \bar{D} & =\frac{\partial}{\partial \theta} .
\end{aligned}
$$

The commutators of these superderivatives and supercharges are given by

$$
\{D, \bar{D}\}=-\frac{\partial}{\partial \tau}, \quad\{Q, \bar{Q}\}=\frac{\partial}{\partial \tau} .
$$

Now we can define an superspace action $S=S_{1}+S_{2}+S_{3}$, where

$$
\begin{aligned}
S_{1} & =\int d^{4} x e d \bar{\theta} d \theta \bar{D} \bar{\Omega} D \Omega \\
& =\int d^{4} x e\left[\bar{c}_{1} \frac{\partial c_{1}}{\partial \tau}+\bar{F} F+i \bar{F} \frac{\partial \psi}{\partial \tau}\right], \\
S_{2} & =\int d^{4} x e d \bar{\theta} d \theta \bar{D} \Omega D \bar{\Omega} \\
& =\int d^{4} x e\left[\bar{c}_{2} \frac{\partial c_{2}}{\partial \tau}+F \bar{F}+i \bar{F} \frac{\partial \bar{\psi}}{\partial \tau}\right], \\
S_{3} & =\int d^{4} x e d \bar{\theta} d \theta S[\bar{\Omega}, \Omega] \\
& =\int d^{4} x e\left[\bar{F} \frac{\delta S_{3}}{\delta \bar{\psi}}+\frac{\delta S_{3}}{\delta \psi} F+\bar{c}_{1} \frac{\delta^{2} S_{3}}{\delta \psi \delta \bar{\psi}} c_{1}+\bar{c}_{2} \frac{\delta^{2} S_{3}}{\delta \psi \delta \bar{\psi}} c_{2}\right] .
\end{aligned}
$$

It may now be noted that this action $S$ coincides with the component action, if we make the following changes of variables $\bar{F} K^{-1} \rightarrow \bar{F}, \bar{\psi} K^{-1} \rightarrow \bar{\psi}, \bar{c}_{1} K^{-1} \rightarrow$ $\bar{c}_{1}, \bar{c}_{2} K^{-1} \rightarrow \bar{c}_{2}$, and $F \rightarrow F, \psi \rightarrow \psi, c_{1} \rightarrow c_{1}, c_{2} \rightarrow c_{2}$ 61.

Thus, we have performed stochastic quantization of the Lifshitz fermionic theory based generalized uncertainty principle using superspace formalism. It may be noted that stochastic quantization of this system induces a supersymmetry corresponding to the extra fictitious time variable. 


\section{Conclusion}

In this paper we deformed the Lifshitz field theories to make them consistent with the existence of a minimum measurable length. This was done by incorporating generalized uncertainty principle in them. We had to promote a parameter used in the theory to a background field with interesting scaling properties, to preserve the Lifshitz scaling of the deformed theory. We also analysed a deformed Lifshitz theory gauge theory based on the generalized uncertainty principle. We observed that even though this theory is non-local, it is invariant under local gauge transformations. We are expect to obtain similar results, if we generalize this work by incorporating terms linear in the momentum, in the deformed Heisenberg algebra 62 - 66. It would also be interesting to analyse the BRST symmetry for this theory. We also performed the stochastic quantization of the deformed Dirac equation semi-classically. It may be noted that the a deformed version of the general relativity has been obtained based on generalized uncertainty principle [22]. It would be interesting to analyse the effect a combination of Lipschitz scaling and generalized uncertainty principle can have on general relativity.

The holographic dual to the Lifshitz field theory has also been studied 67. [71. The dual of the field theory vacuum has a bulk metric,

$$
d s^{2}=-r^{2 z} d t^{2}+r^{2} d x^{2}+L^{2} r^{-2} d r^{2},
$$

where $L^{2}$ represents the overall curvature scale. It is obvious that for $z=1$, this metric reduces to the usual $A d S$ metric. In these Lifshitz theories, the renormalization group flow at finite temperature is used for evaluating the dependence of physical quantities such as the energy density on the momentum scale [72]. Furthermore, the holographic renormalization of gravity in asymptotically Lifshitz spacetimes naturally reproduces the structure of gravity with anisotropic scaling 733. The holographic counter-terms induced near anisotropic infinity take the form of the action for gravity at a Lifshitz point, with the appropriate value of the dynamical critical exponent. The holographic renormalization of Horava-Lifshitz gravity reproduces the full structure of the $z=2$ anisotropic Weyl anomaly in dual field theories in three dimensions [74. In fact, Lifshitz theories have also become important because of the development of HoravaLifshitz gravity [75-79. Horava-Lifshitz gravity is a renormalizable theory of gravity, in which unitarity is not spoiled. Even though gravity is not renormalizable, it can be made renormalizable by adding higher order curvature terms to it. However, the addition of higher order temporal derivatives spoils the unitarity of the theory. A way out of this problem is to add higher order spatial derivatives without adding any higher order temporal derivatives. Even though this break Lorentz symmetry, the Horava-Lifshitz theory of gravity reproduces General Relativity in the infrared limit.

It may be noted that the string theory comes naturally equipped with a minimum measurable length scale, which is the string length scale. This is because the spacetime cannot be probed below this scale 80. Furthermore, the existence of a minimum measurable length scale in a theory produces higher derivative corrects terms, due to the existence of generalized uncertainty principle 81. The the $C F T$ dual to a massive free scalar field theory with such higher derivative corrections has been analysed using the $A d S / C F T$ correspondence 
82. So, it will be interesting to analyse the Lifshitz deformation of $A d S / C F T$ correspondence, consistent with generalized uncertainty principle.

As we have both the fermionic and bosonic actions, it will be interesting to analyse supersymmetric theories based on such deformations. This can be done by constructing various supersymmetric theories based on generalized uncertainty principle with Lifshitz scaling. It may be noted that Lifshitz supersymmetric theories have already been constructed 83 . In fact, according to $A d S / C F T$ correspondence type $I I B$ superstring on $A d S_{5} \times S^{5}$ is dual to the maximally supersymmetric $\mathcal{N}=4$ super-Yang-Mills theory in four dimensions [84-87], so, this result will can be used to study the gravity dual to such a theory. Furthermore, as $A d S_{5} \times S^{5} \sim S O(2,4) / S O(1,4) \times S O(6) / S O(5) \subset$ $S U(2,2 \mid 4) / S O(1,4) \times S O(5)$, so, the superisometries of this background are generated by the supergroup $S U(2,2 \mid 4)$, which also generates the superconformal invariance of $\mathcal{N}=4$ super-Yang-Mills theory in four dimensions. Here the four dimensional superconformal transformations are generated by $S O(2,4)$ and the $R$-symmetry is generated by $S O(6) \sim S U(4)$. Furthermore, $\mathcal{N}=4$ superYang-Mills theory, with $U(N)$ as the gauge group, is the low-energy limit for a stack of multiple coincident D3-branes on $A d S_{5} \times S^{5}$. Here the transverse D3brane coordinates give rise to six scalar fields in the $\mathcal{N}=4$ super-Yang-Mills theory. Apart from these six bosons, there are also sixteen fermions. Thus, a Lifshitz deformation of bulk theory, consistent with generalized uncertainty, may produce interesting deformation of the $\mathcal{N}=4$ super-Yang-Mills theory. It will be interesting to analyse such a deformed super-Yang-Mills theories.

\section{Acknowledgement}

We would like to thank Ali Nassar for pointing out to us an interesting technique used in conformal field theories, i.e., the parameters in a conformal field theory can be promoted to background fields. These background fields can have interesting scaling properties.

\section{References}

[1] D. Amati, M. Ciafaloni and G. Veneziano, Phys. Lett. B 216, 41 (1989)

[2] A. Kempf, G. Mangano, and R. B. Mann, Phys. Rev. D 52, 1108 (1995)

[3] L. N. Chang, D. Minic, N. Okamura, and T. Takeuchi, Phys.Rev. D65, $125027(2002)$

[4] L. N. Chang, D. Minic, N. Okamura, and T. Takeuchi, Phys. Rev. D65, $125028(2002)$

[5] S. Benczik, L. N. Chang, D. Minic, N. Okamura, S. Rayyan, and T. Takeuchi, Phys. Rev. D66, 026003 (2002)

[6] P. Dzierzak, J. Jezierski, P. Malkiewicz, and W. Piechocki, Acta Phys. Polon. B41, 717 (2010)

[7] D. Amati, M. Ciafaloni, and G. Veneziano, Phys. Lett. B 216, 41 (1989) 
[8] M. Maggiore, Phys. Lett. B 304, 65 (1993)

[9] M. Maggiore, Phys. Rev. D 49, 5182 (1994)

[10] M. Maggiore, Phys. Lett. B 319, 83 (1993)

[11] L. J. Garay, Int. J. Mod. Phys. A 10, 145 (1995)

[12] F. Scardigli, Phys. Lett. B 452, 39 (1999)

[13] C. Bambi, F. R. Urban, Class. Quantum Grav. 25, 095006 (2008)

[14] K. Nozari, Phys. Lett. B. 629, 41 (2005)

[15] K. Nozari, T. Azizi, Gen. Relativ. Gravit. 38, 735 (2006)

[16] P. Pedram, Int. J. Mod. Phys. D 19, 2003 (2010)

[17] A. Kempf, G. Mangano, and R. B. Mann, Phys. Rev. D 52, 1108 (1995)

[18] A. Kempf, J. Phys. A 30, 2093 (1997)

[19] F. Brau, J. Phys. A 32, 7691 (1999)

[20] K. Nozari, and B. Fazlpour, Chaos, Solitons and Fractals, 34, 224 (2007)

[21] S. Das, and E. C. Vagenas, Phys. Rev. Lett. 101, 221301 (2008)

[22] M. Kober, Phys. Rev. D 82, 085017 (2010)

[23] V. Husain, D. Kothawala and S. S. Seahra, Phys. Rev. D 87, 025014 (2013)

[24] M. Kober, Int. J. Mod. Phys. A 26, 4251 (2011)

[25] R. M. Hornreich, M. Luban and S. Shtrikman, , Phys. Rev. Lett. 35, 1678 (1975)

[26] G. Grinstein, Phys. Rev. B 23, 4615 (1981)

[27] P. M. Chaikin and T. C. Lubensky, Principles of Condensed Matter Physics, Cambridge University Press, Cambridge, UK (1995)

[28] S. Sachdev, Quantum Phase Transitions, Cambridge University Press, Cambridge, UK (2001)

[29] A. Benlagra and M. Vojta, Phys. Rev. B 87, 165143 (2013)

[30] M. Bercx and F. F. Assaad, Phys. Rev. B 86, 075108 (2012)

[31] P. Gegenwart, Nature Phys. 4, 186 (2008)

[32] P. Coleman, C. Pepin, Q. Si and R. Ramazashvili, J. Phys. Condens. Matt. 13, R723 (2001)

[33] A. Hackl and M. Vojta, Phys. Rev. Lett. 106, 137002 (2011)

[34] V. B. Svetovoy, Phys. Rev. Lett. 101, 163603 (2008)

[35] S. A. Ellingsen, I. Brevik, J. S. Hoye and K. A. Milton, Phys. Rev. E 78, $021117(2008)$ 
[36] M. Bordag, B. Geyer, G. L. Klimchitskaya and V. M. Mostepanenko, Phys. Rev. B 74, 205431 (2006)

[37] M. Alishahiha, M. R. M. Mozaffar and A. Mollabashi, Phys. Rev. D 86, $026002(2012)$

[38] M. Liu, J. Lu and J. Lu, Class. Quant. Grav. 28, 125024 (2011)

[39] H. Montani and F. A. Schaposnik, Phys. Rev. D 86, 065024 (2012)

[40] L. Caffarelli and L. Silvestre, Comm. Part. Diff. Eqs. 32, 1245 (2007)

[41] R. T. Seeley, Proc. Symp. Pure Math. 10, 288 (1967)

[42] C. Laemmerzahl, J. Math. Phys. 34, 3918 (1993)

[43] J. J. Giambiagi, Nuovo Cim. A 104, 1841 (1991)

[44] C. G. Bollini and J. J. Giambiagi, J. Math. Phys. 34, 610 (1993)

[45] D. Anselmi and M. Halat, Phys. Rev. D 76, 125011 (2007)

[46] D. Anselmi, Eur. Phys. J. C 65, 523 (2010)

[47] A. Dhar, G. Mandal and S. R. Wadia, Phys. Rev. D 80, 105018 (2009)

[48] Z. Komargodski, JHEP. 1207, 069 (2012)

[49] Z. Komargodski, JHEP 1112, 099 (2011)

[50] J. Tan, Calc. Var 42, 21 (2011)

[51] G. Parisi and Y. S. Wu, Sci. Sin. 24, 483 (1981)

[52] A. Barchielli and G. Lupieri, J. Math. Phys. 26, 2222 (1985)

[53] F. Guerra and R. Marra, Phys. Rev. D29, 1647 (1984)

[54] P. H. Damgaard and K. Tsokos, Nucl. Phys. B 235, 75 (1983)

[55] P. H. Damgaard and H. Huffel, Phys. Rep. 152, 227 (1987)

[56] G. Parisi and N. Sourlas, Nucl. Phys. B 206, 321 (1982)

[57] L. Henriet, Z. Ristivojevic, P. P. Orth and K. Le Hur, Phys. Rev. A 90, 023820 (2014)

[58] U. Lindstrom, M. Rocek, W. Siegel, P. van Nieuwenhuizen, and A. E. van de Ven, J. Math. Phys. 31, 1761 (1990)

[59] G. Parisi and N. Sourlas, Nucl. Phys. B 206, 321 (1982)

[60] S. Chaturvedi, A. K. Kapoor and V. Srinivasan, Phys. Lett. B 140, 56 (1984)

[61] H. Montani and F. A. Schaposnik, Phys. Rev. D86, 065024 (2012)

[62] A. F. Ali, S. Das and E. C. Vagenas, Phys. Rev. D84, 044013 (2011) 
[63] P. Pedram, K. Nozari and S. H. Taheri, JHEP 1103, 093 (2011)

[64] M. Asghari, P. Pedram and K. Nozari, Phys. Lett. B 725, 451 (2013)

[65] S. Das, E. C. Vagenas and A. F. Ali, Phys. Lett. B 690, 407 (2010)

[66] W. Chemissany, S. Das, A. F. Ali and E. C. Vagenas, JCAP. 1112, 017 (2011)

[67] J. M. Maldacena, Adv. Theor. Math. Phys. 2, 231 (1998)

[68] S. Kachru, X. Liu and M. Mulligan, Phys. Rev. D78, 106005 (2008)

[69] K. Balasubramanian and K. Narayan, JHEP. 1008, 014 (2010)

[70] R. Gregory, S. L. Parameswaran, G. Tasinato and I. Zavala, JHEP. 1012, $047(2010)$

[71] A. Donos and J. P. Gauntlett, JHEP. 1012, 002 (2010)

[72] M. Park and R. B. Mann, JHEP. 1207, 173 (2012)

[73] T. Griffin, P. Horava and C. M. M. Thompson, JHEP. 1205, 010 (2012)

[74] T. Griffin, P. Horava and C. M. M. Thompson, Phys. Rev. Lett. 110, 081602 (2013)

[75] P. Horava, Phys. Lett. B 694,172 (2010)

[76] P. Horava, Phys. Rev. D 79, 084008 (2009)

[77] P. Horava, JHEP. 03, 020 (2009)

[78] O. Obregon and J. A. Preciado, Phys. Rev. D 86, 063502 (2012)

[79] A. Sheykhi, Phys. Rev. D 87, 024022 (2013)

[80] D. Amati, M. Ciafaloni and G. Veneziano, Phys. Lett. B 216, 41 (1989)

[81] S. Das and E. C. Vagenas, Phys. Rev. Lett. 101, 221301 (2008)

[82] M. Faizal. A. F. Ali and A. Nassar, arXiv:1405.4519 (2014)

[83] M. Pospelov and C. Tamarit, JHEP 01, 048 (2014)

[84] O. Aharony, S. S. Gubser, J. M. Maldacena, H. Ooguri and Y. Oz, Phys. Rept. 323, 183 (2000)

[85] E. Witten, Adv. Theor. Math. Phys. 2, 253 (1998)

[86] J. M. Maldacena and C. Nunez, Phys. Rev. Lett. 86, 588 (2001)

[87] A. Karch and E. Katz, JHEP. 0206, 043 (2002) 